\title{
On the intensity of the radiation of gas under the influence of the electrical discharge
}

\section{K. Ångström}

To cite this article: K. Angström (1892) On the intensity of the radiation of gas under the influence of the electrical discharge, Philosophical Magazine Series 5, 33:203, 387-391, DOI: 10.1080/14786449208620009

To link to this article: http://dx.doi.org/10.1080/14786449208620009

曲 Published online: 08 May 2009.

Submit your article to this journal $\sqsubset \pi$

Џ Article views: 2

Q View related articles $\square$ 
southern part of the island, and layers of volcanic dust occur in it at various horizons. There is everywhere a passage from the more siliceous to the more calcareous earths.

From the palæontological and lithological evidence the authors conclude that the depth of water in which the 0ceanie beds were deposited varied between 1000 and 2500 fathoms. The microscopical and chemical evidence shows that the Radiolarian earths are similar to modern Radiolarian ooze ; that the calcareo-siliceous earths are similar to what is called by Prof. Haeckel "mixed Radiolarian ooze"; that some of the Foraminiferal earths are comparable to Globigerina-ooze from 1000 fathoms, and that others greatly resemble European Chalk; and, finally, that the coloured clays bear a strong resemblance to the so-called "red clays" of modern oceanic areas. Hence the raised Oceanic deposits of Barbados seem to present us with an epitome of the various kinds of deposits which are found on the floors of warm seas at the present day. Equivalent deposits are known in Trinidad and Jamaica; and it is inferred by the authors that the whole Central American and Caribbean region was deeply submerged during the Pliocene period, leaving free communication at that time between the Atlantic and Pacific Oceans.

An Appendix by Mr. W. Hill treats of the minute structure of the Oceanic earths and limestones and of the Foraminiferal muds and detrital earths; and this is supplemented by a Report from Miss Raisin on the inorganic material of certain Barbados rocks.

3. "Archceopneustes abruptus, a new Genus and Species of Echinoid from the Oceanic Series in Barbados." By J. W. Gregory, Esq., B.Sc., F.G.S.

\section{Intelligence and Miscellaneous Articles.}

\section{ON THE INTENSITY OF THE RADIATION OF GAS UNDER THE INFLUENCE OF THE ELECTRICAL DISCHARGE. PRELIMINARY NOTICE BY K. АNGSTRöM *.}

I. THE interesting phenomena which accompany electrical discharge through rarefied gases, although still for the most part unexplained, have of late been repeatedly investigated both in the optical and in the electrical direction. The quantitative relation between the optical and the electrical phenomena has, however, been but little studied. Now it is only by a more perfect knowledge of this relationship that we may hope to understand and explain the origin and the nature of the radiation which we observe in Geissler's tubes, as also the part which electricity plays in those remarkable phenomena.

Calorimetrical researches on the development of heat in Geissler's

* Tran sla ted from a separate impression communicated by the Author. 
tubes have been made by E. Wiedemann (Wied. Annalen, vol. vi. p. 298, 1879, and vol. x. p. 202, 1880), and by M. Nasselberg (Mém. de l'Acad. Imp. des sciences de St. Pétersbourg, vol. vi. No. 1, 1879). M. G. Staub further has recently (Inaugural dissertation, 2nd part, Zurich, 1890) made some experiments on the development of heat in the discharge of Leyden jars through Geissler's tubes filled with air and hydrogen : he has at the same time attempted to determine the ratio of the obscure to the luminous radiation. With this view he enclosed the tube in a Bunsen's ice-calorimeter, where the tube and the calorimeter successively transmitted or absorbed the luminous radiation; in the latter case the tube was blackened.

Not only the total energy absorbed by the gas and that radiated have been measured in these experiments, but also the energy received directly from the sides of the tubes, and which they changed into heat. However valuable these researches may have been, they do not give a direct answer to the question of the radiation of the gas under the action of the electric discharge. A direct determination of this intensity has not yet been made so far as I know, and the possibility of such a determination, with our present resources, has even been questioned. For this reason I bave endeavoured to use the bolometer to determine the radiation of different gases under the action of the electrical current; to express the intensity of this radiation in absolute measure; and to endeavour then to determine the relation of the radiation to the causes which produce it.

After having worked for more than a year at this very arduous investigation, I think I may consider that the preliminary trial experiments are completed. I shall explain here some of the results at which I have arrived ; treating afterwards with greater detail the materials which I have collected.

II. As the light of the kathode is too feeble and its shape too variable to justify the hope that I could obtain very accurate quantitative results, I restricted myself to the investigation of the positive light only.

I used cylindrical glass tubes of the same thickness and 10 to $15 \mathrm{~mm}$. in diameter. The electrodes were usually placed in side tubes at right angles with the principal tube. One end of the tube and sometimes both were closed by a smooth plate of rocksalt fixed hermetically by means of silicate of soda. In the prolongation of the axis of the tube a sensitive bolometer was placed, separated from the tube by a double screen with apertures in the direction of the axis of the tube. Between the sides of the screen was another double movable screen.

'The indications of the bolometer were compared with one of $\mathrm{my}$ instruments for determining radiant heat in absolute measure (Acta Reg. Soc. Upsal. series iii. 1886. See also Öfversigt af Kongl. Vet.-Akad. Forhandl. No. 6, p. 379, 1888). I found in this way that one division of the scale corresponded to a radiation of $278 \times 10^{-9}$ gramme calories per second and square centimetre. 
To ascertain if and how far the character of the radiation changes with the different conditions of the gas, I measured on the one hand the total radiation, and on the other the radiation transmitted by a plate of alum of $3.95 \mathrm{~mm}$. in thickness. The radiation determined by direct observations consists of two parts-the radiation of the gas, and that of the more or less heated sides of the tube. The latter part must be eliminated, which is effected by a series of observations of the radiation of the tube after the current has been opened. From the curve of radiation thus found it is easy to calculate the radiation of the tube during the experiment, and by subtracting this quantity from the quantity observed during the passage of the current the radiation of the gas is obtained. This value, however, is increased by the reflexion of the sides of the discharge-tube, and diminished on the other hand by the reflexion of the plate of rock-salt which closes the tube. Direct experiments, the details of which I suppress, have enabled me to make a satisfactory correction for this reflexion, and therefore to determine the total radiation in absolute measure.

As source of electricity I principally used an accumulator of 800 elements of Planté's pattern, and also a Rubmkorff's coil of medium size. The current was determined by a dead-beat reflectinggalvanometer, with a wire insulated by caoutchouc. The diference of potential between two points in the positive light was measured by means of two thin platinum wires, soldered in the discharge-tube, and connected with a Mascart's quadrant-electrometer. For exhausting the tube I used, in my later experiments, a pump on Sprengel's principle, modified by Prytz (Wiedemann's Annalen), and further improved by myself.

I have spared no pains to make the gases as pure as possible. Thus neither stopcocks, nor caoutchouc tubes, nor grease were used either in the preparation of the gas or in the connexion of the tube with the air-pump. To introduce the gases into the discharge-tube, to dry them, and to prevent the access of mercurial vapour to the tube, I used the method of M. Cornu (Journal de Physique), slightly modified for my purpose. My researches extended to oxygen and hydrogen liberated by electrolysis of pure water acidulated by phosphoric acid; to nitrogen produced by leading pure air freed from carbonic acid over heated copper (first reduced by hydrogen); and to carbonic acid produced by heating sulphuric and oxalic acids and purified by passing through a solution of potash.

III. I give here the most important results of this research.

1. For a given pressure, the radiation of positive light is proportional to the intensity of the principal current. It is true that on using very powerful currents small divergences are observed, but in this case they are always accompanied by a permanent alteration of the gas, so that the same radiation is not met with again for less strong currents.

2. If the electric current is kept constant and the pressure is varied, it appears that the intensity of total radiation scarcely varies 
between the limits 0.1 to $1.5 \mathrm{~mm}$., but it increases a little at higher pressures. At pressures exceeding $1.5 \mathrm{~mm}$. the character of the discharge seems to be modified, after which the radiation increases considerably with the pressure after having passed, at any rate in certain cases, through a minimum.

3. For the same gas under the same pressure the composition of the radiation is constant, and does not depend on the intensity of the electrical current. This is subject to the reserve stated in (1).

4. When the density of the gas changes the composition of the radiation changes also, in so far that the ratio of the intensity of radiation of the shorter wave-lengths to that of the total radiation decreases with increase of pressurt.

This ratio, indicated in my experiments by the ratio of the radiation transmitted by a plate of alum to that of the total radiation, varies for instance between the pressures $0.1-1.6 \mathrm{~mm}$., for carbon dioxide from 46 to 15 per cent, and for nitrogen from 94 to 60 per cent.

5. It will be seen from these figures that the ratio between the luminous and the total radiation rises to considerable values for very low pressures, values far greater than those of our ordinary luminous sources. This ratio, however, does not always attain such high values in the electrical discharge in rarefied gases. At pressures of $5-10 \mathrm{~mm}$. it is rather small.

6 . The intensity of the total radiation varies greatly for different gases. It is not in a simple ratio with the molecular weight of the substance, or with the difference of potential in the layer of radiating gas. It does not appear to depend essentially on the absorption of the gas at the ordinary pressures and temperatures, either as regards its intensity or in reference to its composition, as can be seen by the following table, which contains values for the radiation and the difference of potential for a pressure of $0.7 \mathrm{~mm}$. and for the same strength of the electrical current.

\begin{tabular}{|c|c|c|c|}
\hline & Total. & $\begin{array}{c}\text { Radiation } \\
\text { transmitted } \\
\text { by alum. }\end{array}$ & $\begin{array}{c}\text { Difference } \\
\text { of potential. }\end{array}$ \\
\hline Oxygen .............. & $1 \cdot 0$ & $\ldots \ldots$ & 17 \\
Eydrogen ............. & 5.9 & $2 \cdot 2$ & 19 \\
Nitrogen ............. & $30 \cdot 0$ & $2 \cdot 4$ & 25 \\
Carbon dioxide........ & 13.6 & $3 \cdot 8$ & 27 \\
\hline
\end{tabular}

IV. In the case of low pressures it appears to me that we may give the following explanation of these phenomena.

When the rarefied gas becomes a conductor of an electrical current, it is the molecules principally which serve to transport electricity, that is to say the active molecules which radiate. The 
number of these molecules is proportional to the intensity of the current, and varies but little with the variations of pressure as long as the discharge retains its character (III. 1 and 2). This radiation is of an irregular character according to M. R. von Helmholtz (Die Licht und Wärmestrahlung verbrennender Gase, Berlin 1890); or it belongs to the class of phenomena to which M. E. Wiedemann (Wied. Ann. xxxvii. p. 177, 1889) has given the name of phenomena of luminescence in this sense, that the composition of the radiation does not correspond with the temperature of the gas. The radiation may be of extreme intensity in the groups of short wavelengths.

When the pressure increases and with it the number of inactive molecules, the irregular radiation of the active molecules may more easily be transmitted to the former. The number of radiating molecules increases therefore, and consequently also the radiation of the gas, but it changes in character in this sense, that the radiation of the groups of shorter wave-lengths relatively diminishes.Öfversigt af Kongl. Akad. Förhandl., 1891, No. 6.

\section{ON THE REPULSIVE FORCE OF RADIATING BODIES. BY PETER LEBEDEW.}

It was shown by Maxwell * that a pressure is exerted on absorptive bodies by incident rays in the direction of their propagations, and that this can be expressed in the form

$$
\mathrm{P}=\frac{\mathrm{E}}{v}, \cdot \cdot \cdot \cdot \cdot \cdot \cdot \cdot \cdot
$$

in which $\mathbf{E}$ is the energy imparted to the body by the incident rays in unit time, and $v$ the velocity of light in the medium in which the body is placed.

Independently of Maxwell, Bartoli $\dagger$ and Boltzmann $\ddagger$ arrived at analogous results in treating the case of reflexion. Bartoli found that at perpendicular incidence the rays exert a pressure against a mirror, which is twice as much as that which, according to Maxwell, they would exert against an absorbent body.

In. what follows the ratio of the repulsion produced by radiation to the Newtonian attraction is deduced botb for our sun, and for a hot spherical body in general. The expressions obtained hold only for absolutely black bodies, whose dimensions are great in comparison with the wave-length of the incident radiation; accordingly the interesting questions respecting the repulsive force in the tails of comets, and the mutual action of two adjacent molecules in the body cannot be solved, but only indicated.

As it is only the order of the magnitude of the desired ratio

* Electricity and Magnetism, \$792.

† Exner's Repertorium, vol. xxi. p. 198 (1885).

† Wied. Ann. vol. xxii. p. 31 (1884). 